\title{
MANGANESE AND CARBON LINES AS TEMPERATURE INDICATORS
}

\author{
G. ELSTE \\ Department of Astronomy, University of Michigan, Ann Arbor, MI 48109, U.S.A.
}

(Received 4 June; in revised form 1 October, 1986)

\begin{abstract}
Selected hyperfine structure broadened lines of various excitations and the carbon line at $5380 \AA$ are tested as temperature diagnostics for photospheric heterogeneities. This is done by comparing the observed center-to-limb variation of the equivalent widths of these lines with predictions by several proposed models of homogeneous type. Model 10 (Elste, 1968), which also explains the limb-darkening of the continuum in the same spectral range, provides a better basis for the analysis of heterogeneities at different photospheric levels than other recent reference models.
\end{abstract}

\section{Introduction}

Pure temperature indicators, possibly more sensitive than the continuum intensity, are required for mapping temperature differences in the solar granulation at various depths (Elste, 1985a, b). They are also useful for measuring time-variations of the average temperature of the Sun as a star as done by Livingston (1985). Most spectral lines of intermediate strength, however, are poorly suited for that purpose since their total absorption depends on the broadening of the line absorption coefficient by non-thermal motions of small scale. This so-called microturbulence then constitutes another variable. For example with approach of the Sun's limb a larger non-thermal broadening is produced by the averaging over an extended geometrical length containing different parts of the convective velocity field. There are, however, spectral lines of certain elements, for which the line-absorption coefficient is intrinsically broadened due to the interaction of the electron shell with the nuclear spin, i.e., by hyperfine structure splitting. If this is sufficiently wide the variation of an additional broadening by non-thermal motions will be negligible. Mn I 5395 and 5420 were first used by Elste and Teske (1977) for analyzing the photospheric network structure. In addition to a consideration of the above-mentioned lines, which mostly originate in the middle photosphere, we should also look at lines which form as deep as possible in order to obtain information about temperature gradients. It is the combination of equivalent widths of lines formed in deep and in intermediate layers, which will permit the determination of temperature gradients in photospheric fine structure.

The present investigation wishes to test the temperature sensitivity of selected lines. This can most efficiently be done by comparing the observed center-to-limb variation of their equivalent widths with that predicted by a standard model atmosphere. While line blending can easily be noticed in precision line profiles, subtle effects of the temperature stratification and severe departures from Local Thermodynamic Equilibrium only show up in the center-to-limb variation of the equivalent widths. It will be 
shown that a combination of two selected lines is very well suited to measure temperature gradients. However, it becomes clear that it is not the more recent homogeneous model photosphere by Vernazza et al. (1976), (VAL.M hereafter), which explains the observations best, but one of the older models. The details will be discussed in Section 6 .

The spectrum synthesis for some representative model atmospheres will also supply information about the approximate depth of line formation, which is important for the interpretation of the results obtained with these lines. For the two best suited lines, Ci 5380 and Mn I 5395, the sensitivity to temperature as well as temperature gradients with optical depth are derived in Section 3.

\section{Choice of Lines}

The widest hyperfine structure splitting is found in the lines of neutral manganese. Starting from the ground level, the lines 5394.7 and 5432.5 possess the greatest temperature sensitivity and are relatively clear of blends. They also are sufficiently weak, so that damping by van der Waals interaction and quadratic Stark effect are of minor importance. The medium-excitation $(2.13 \mathrm{eV})$ line 5420.2 is selected because of its large hyperfine structure illustrated in Figure 1. For covering a greater range in depth we wish to add a manganese line of high excitation in the same spectral region. The line 5399.5 was chosen, being the least disturbed of the lines in multiplet No. 42, with $3.84 \mathrm{eV}$ excitation.

Since there are no hyperfine structure broadened lines originating in the deep photosphere, our best choice is one of the high excitation lines of carbon, which is mostly
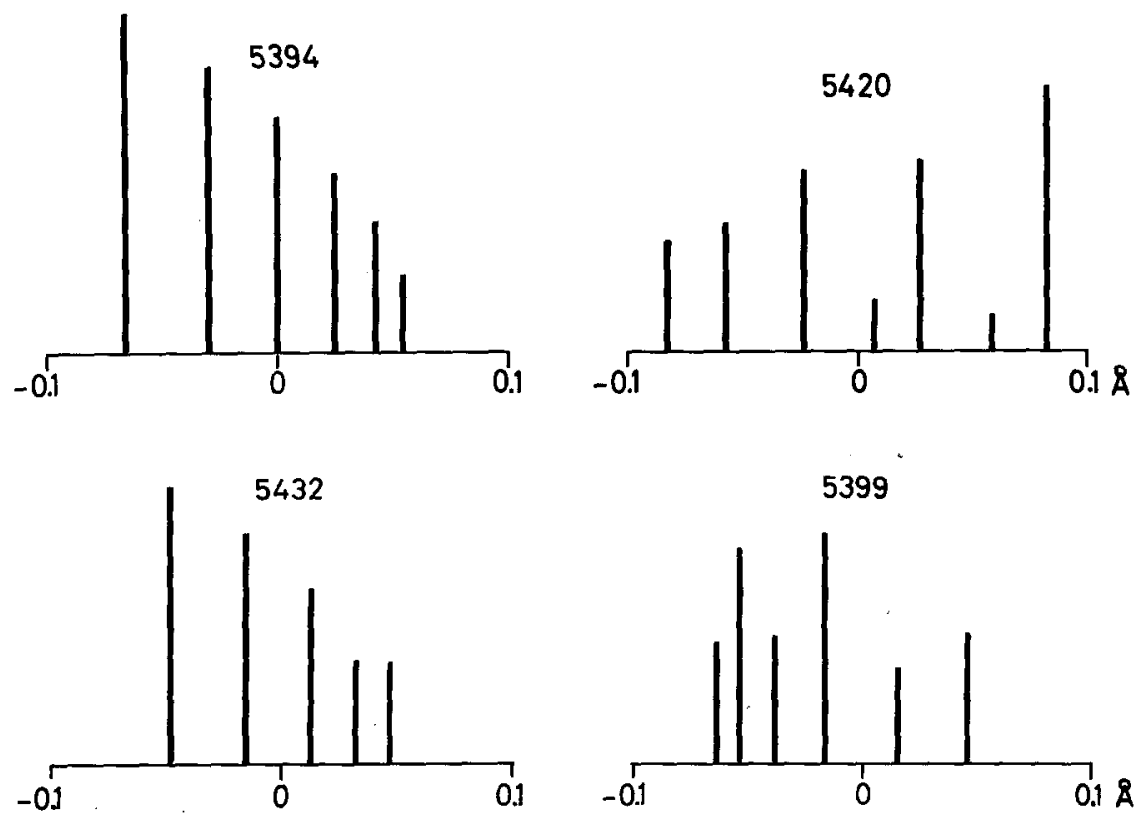

Fig. 1. Hyperfine structure pattern of manganese lines. 
in the neutral state. The thermal broadening of carbon is sufficiently large so that uncertainties in the non-thermal motions are not a major problem. The lines 4770 and 5039 are poorly blended, 5052 is not as clean as 5380 and is also somewhat stronger, making it more sensitive to microturbulence. It would be formed not as deep as 5380 . Another useful line would be 6587 , but its wavelength difference with respect to the 5400 A region would require a correction for differential refraction during early morning hours of good seeing. So we selected 5380 with an excitation potential of the lower level of $7.68 \mathrm{eV}$.

\section{Temperature Sensitivity of C 5380 and Mn 5395}

For mapping the temperature structure in photospheric heterogeneities the lines CI 5380 and $\mathrm{MnI}$ 5395, are useful. They span the depth range of the photosphere in which we expect differences, but avoid contributions from the upper photosphere and lower chromosphere.

We first estimate the temperature dependence of these lines. Generally, equivalent widths of spectral lines depend on the ratio of the line- to continuous absorption coefficient at the depth of line formation. This must then be multiplied by a factor which is the slope of the curve-of-growth for the proper strength of the line. For the following estimate we neglect the influence of the partition functions. In the case of manganese most atoms are singly ionized at photospheric temperatures, so that roughly

$$
x_{l} / x_{\mathrm{H}^{-}} \sim 10^{\left(x_{0}-\chi_{\mathrm{H}-}\right) \Theta},
$$

where $\Theta=5040 / T, \chi_{0}=7.43 \mathrm{eV}$ is the ionization potential of manganese, and $\chi_{\mathbf{H}^{-}}=0.75 \mathrm{eV}$ is the ionization potential of the negative hydrogen ion. Most carbon is neutral, leading to

$$
x_{l} / x_{\mathrm{H}^{-}} \sim 10^{-\left(x_{0, s}+\chi_{\mathrm{H}^{-}}\right) \Theta} \Theta^{-5 / 2} P_{e},
$$

where $\chi_{0, s}=7.68 \mathrm{eV}$ is the excitation potential of the carbon line. In the following evaluation we use values of $\Theta$ and $\log P_{e}$ from Model 10, taken at the approximate depth of formation as calculated with our spectrum synthesis. As can be seen from Figure 3 the manganese line is formed at $\log \tau_{0.5}=-1.6$ with $\Theta=1.056$, while the carbon line, originating at unit optical depth, needs $\Theta=0.784$ and $\log P_{e}=1.787$.

We first discuss models hotter and cooler than Model 10 by $\Delta \Theta=0.005$ at all depths. Consequently the electron pressure at the depth where the carbon line is formed becomes higher and lower by $\Delta \log P_{e}=+0.04$ and -0.03 , respectively. Slightly hotter models have a $\Delta \Theta=-0.005$. This decreases the ratio of line-to-continuum absorption for the manganese line by $\Delta \log \left(x_{\ell} / x_{H^{-}}\right)=-0.0334$, while it increases that for the carbon line by +0.0195 . With values of the slope of the curve-of-growth $\mathrm{d} \log W / \mathrm{d} \log N=0.64$ and 0.73 for the manganese and carbon line, respectively, we expect a weakening of the manganese line by $\Delta \log (W / \lambda)=-0.021$ and a strengthening of the carbon line by +0.014 . Precise spectrum synthesis leads to -0.020 and +0.016 , respectively. 
Next we consider a variation of the temperature gradient in the photospheric fine structure. Therefore, two additional models were tested, one with a slightly steeper the other with a slightly flatter gradient. Starting with Model 10 and at $\log \tau_{0.5}=-0.5$ the variation in $\Theta$ with depth for a stepsize of $\Delta \log \tau_{0.5}=0.1$ was changed by 0.001 both inward and outward. Temperatures for steeper and flatter models cross over at $\log \tau_{0.5}=-0.5$. This depth was chosen since it separates the region of line formation of the two lines. A different depth for the crossover can be achieved by combining the result of this model with that of generally hotter or cooler atmospheres discussed above.

For the steeper model we can expect the carbon line to behave like in the hotter model and the manganese line like in the cooler model, i.e., both lines are getting stronger. The spectrum synthesis led to increases for the carbon line by $\Delta \log (W / \lambda)=0.0255$ and for the manganese line by 0.054 . This agrees roughly with what would be expected from the results of the generally hotter and cooler models. The more pronounced effect for the manganese line is due to the larger relative increase in the temperature gradient in the intermediate photosphere.

We have shown that the lines C 5380 and Mn 5395 are well suited in principle to indicate variations in the temperature structure in heterogeneities. It is now necessary to test the lines by comparing the center-to-limb variation of their equivalent widths with that predicted by reference models for the homogeneous photosphere. It appeared feasable to also include in this test the other three manganese lines, thus providing a broader base for judging the models.

\section{Observations}

Photoelectric tracings of the manganese lines have been obtained at the McMath Hulbert Observatory for $\cos \theta=1.0,0.5,0.3$. A special set of data in digitized form was supplied by Testerman (1973), who observed with the McMath telescope of Kitt Peak National Observatory using the main spectrograph in double pass mode. These observations cover $\cos \theta=1.0,0.63,0.4,0.25$, and 0.16 . The carbon line was observed by the author with the same equipment along a polar diameter of the Sun in 1980 . The positions were $\cos \theta=1.0,0.63,0.40,0.25,0.20$ and 0.15 toward the north and south. These observations agree very well with Elste (1955). All equivalent widths have been reevaluated to conform in the truncation procedure with that used in the spectrum synthesis. The scatter of the points in Figure 2 indicates the noise level of the observed equivalent widths. For some positions we have two independent measurements.

\section{Model Atmospheres}

The purpose of this work is a reliability test of the selected lines as temperature indicators. The task is actually twofold, to test the lines, and also to look at the model atmosphere. The solar photosphere is essentially heterogeneous with a horizontal scale generally larger than the vertical. As long as we, therefore, restrict the test to sufficiently small angles we can anticipate that the effects produced by the light beams traveling 

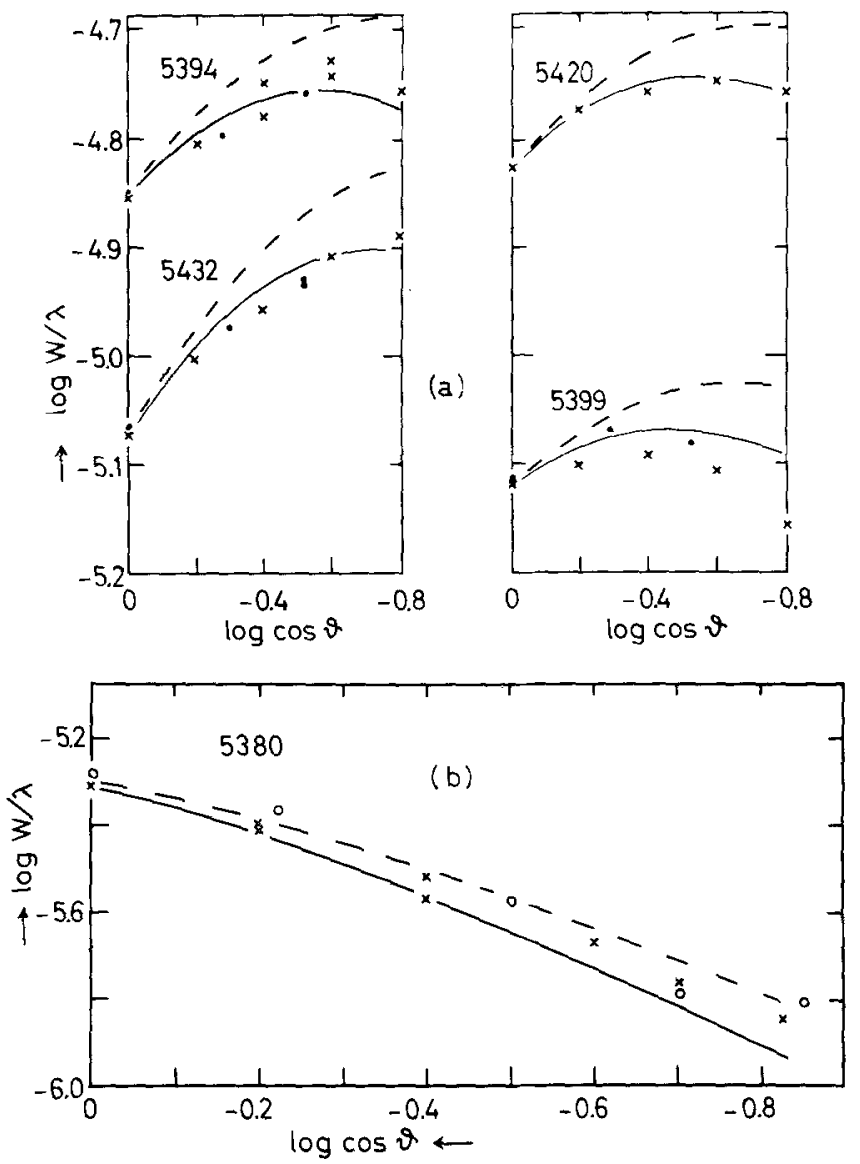

Fig. 2. Center-to-limb variation of equivalent widths. McMath-Hulbert Obs.; $\times$ Kitt Peak; $O$ Göttingen; —— Model 10;--- VAL.M; (a) manganese lines, (b) carbon line.

through various regions of heterogeneities will be of secondary importance. In this case a homogeneous model atmosphere still appears to be a useful approximation; unless we find a contradiction between the model prediction and observations for the limbdarkening of the continuum on one side and for the center-to-limb variation of the lines on the other.

The model representing best the disk brightness temperature as a function of wavelength with less emphasis on the limb-darkening is that by Vernazza et al. (1976). These authors realize the dilemma between absolute energy measurements over an extended wavelength range and the limb-darkening measurements. Absolute measurements are certainly much more sensitive to systematic errors than relative ones. It is clear that heterogeneities affect the center-to-limb variations more with increasing angle of incidence. We must therefore compare the derivative of the quantities to be tested with $(\log \cos \theta)$, taken at disk center.

As a second model we consider Model 10 (Elste, 1968). In the construction of this model, more emphasis was placed on the limb-darkening, permitting larger margins for 
TABLE I

Difference between observed and predicted limb-darkening at $5400 \AA$ and the slope at disk center $\mathrm{d}(\log I) / \mathrm{d}(\log \cos \theta)$

\begin{tabular}{lrrrrrr}
\hline $\cos \theta$ & \multicolumn{1}{c}{0.8} & 0.6 & 0.45 & 0.3 & \multicolumn{1}{c}{0.2} & Slope \\
\hline Model 10 & 0.001 & 0.003 & 0.008 & 0.009 & 0.004 & 0.561 \\
MODFD.10 & -0.001 & 0.000 & 0.004 & 0.006 & 0.002 & 0.551 \\
VAL.M & -0.011 & -0.018 & -0.017 & -0.015 & -0.015 & 0.497 \\
& & & & & Observed: & 0.567
\end{tabular}

the energy distribution at disk center. The Harvard-Smithsonian Reference Atmosphere (Gingerich et al., 1971) is very close to Model 10 in the middle photosphere. In order to possibly improve the stratification in the temperature minimum region Model 10 has been modified to follow VAL.M for $\log \tau_{0.5}<-3.0$. This model is designated MODFD.10. Since the models VAL.M and Model 10 have been described and discussed in detail by Vernazza et al. (1976), and the model MODFD.10 is only a minor variation of Model 10 in the outer layers, we will not go into further details. However, the limb-darkening in the $5400 \AA$ spectral region shall be discussed. Recent observations at Kitt Peak (Petro et al., 1984; Pierce and Slaughter, 1977) are systematically located slightly above previous observations by Pierce at the Snow Telescope (Mt. Wilson) and at McMath-Hulbert Observatory (David and Elste, 1962). But observations with a Gregory-Coudé telescope by Wittmann (1980) support the older observations. We, therefore, decided to rely on the older observations, which already served as a base for Model 10. Table I lists the differences of the observed minus the predicted limb-darkening for the three models. With regard to the influence of heterogeneities the last column gives the derivative of the logarithms of the quantities at disk center. We have chosen $(\log \cos \theta)$ because this quantity is nearly proportional to the geometrical depth of continuum formation. Model 10 describes the limb-darkening behaviour near disk center better than the modified MODFD.10, while VAL.M deviates even more.

\section{Center-to-Limb Variation of the Lines}

The spectrum synthesis of the lines has been carried out in wavelength steps of $15 \mathrm{~mA}$ out to a line depth of 0.0003 . For the manganese lines the line absorption coefficient is the sum of the Voigt profiles with displacements and relative intensities of the components of the particular hyperfine structure pattern. These patterns were calculated according to Brix and Kopfermann (1952). Components, closely spaced with respect to the total splitting, have been combined to reduce the computing effort. The pattern used for each line is displayed in Figure 1. Non-thermal motions of small scale were kept constant with depth at $1 \mathrm{~km} \mathrm{~s}^{-1}$. Depth dependent damping by van der Waals forces and quadratic Stark effect was taken into account according to the formulae given by 
Unsöld (1955). The depth variation of the occupation numbers of the atomic levels were calculated according to the Saha and Boltzmann equations. For the source function the Planck function of the local temperature was used, assuming Local Thermodynamic Equilibrium.

In Figure 2(a) we compare the observed center-to-limb variation of the equivalent widths of the four selected manganese lines with the predictions by Model 10 and VAL.M. We see that Model 10, which already described the continuum better than VAL.M, also provides a better explanation of the equivalent width variation of all these lines. In every case the model VAL.M demands increasingly larger than observed values with approach of the limb. The situation for the deeply formed carbon line is by far not so critical as can be seen in Figure 2(b). As expressed in Section 5, we should restrict our evaluation to small angles of incidence because of the effect of heterogeneities. For that purpose we list in Table II the derivative of $(\log W / \lambda)$ with respect to $(\log \cos \theta)$ at the center of the disk for the observations and for the three models. The uncertainty of the observed derivatives is about 0.02 . The comparison of these values leads to the same conclusion, but it is better founded.

TABLE II

Slope of equivalent width variation with $\cos \theta$ at disk center $\mathrm{d}(\log W) / \mathrm{d}(\log \cos \theta)$

\begin{tabular}{lccccc}
\hline & \multicolumn{2}{l}{ Manganese lines } & & \multirow{2}{*}{ Carbon } \\
\cline { 2 - 5 } & 5395 & 5432 & 5420 & 5399 & 5380 \\
\hline Observed & -0.32 & -0.34 & -0.27 & -0.22 & 0.50 \\
Model 10 & -0.34 & -0.47 & -0.29 & -0.21 & 0.54 \\
MODFD.10 & -0.38 & -0.50 & -0.30 & -0.22 & 0.54 \\
VAL.M & -0.42 & -0.54 & -0.36 & -0.28 & 0.46 \\
\hline
\end{tabular}

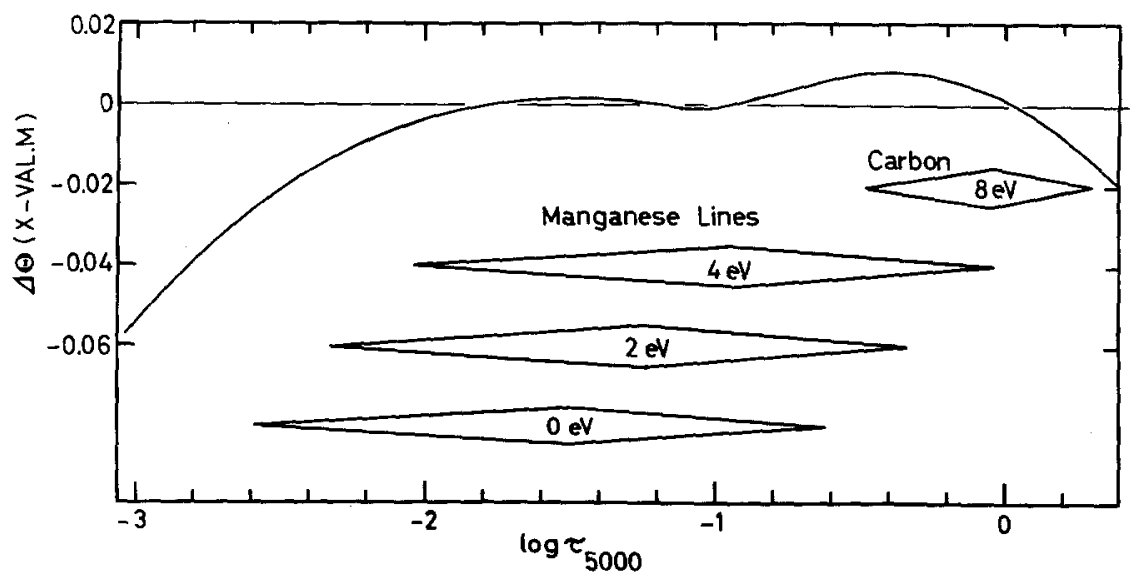

Fig. 3. Difference in $\Theta$ between Model 10 and VAL.M as a function of $\log \tau_{0.5}$ with regions of line formation indicated for various excitations. 
For the interpretation of the results we display in Figure 3 the difference in $\Theta=5040 / T$ between Model 10 and VAL.M as a function of $\log \tau_{0.5}$. We also indicate the depth range for the formation of the lines. This is done by marks, which extend from $50 \%$ above to $50 \%$ below the maximum contribution. Details about these contribution curves can be found in the appendix of the work by Elste and Teske (1977) and in Elste (1969). Since the lines are sufficiently weak, those of high excitation form at greater depths than those of lower excitation. This is not new, but it is mostly not realized that line formation extends over a substantial range in depth. With this information we recognize that the poor explanation of the manganese lines by VAL.M is not just the rather low temperature in the temperature minimum region, but also the different gradient in the depth range in which the continuum is formed. This must be kept in mind for interpreting the differential changes of equivalent widths in the mapping of temperature gradients (Elste, 1985a, b).

\section{Line Profile of Mn 5395}

Since we approximate in this procedure the dynamic solar photosphere by a homogeneous model, the comparison of a line profile with observations has to incorporate the additional broadening by macroscopic motions. The line profile of 5395, as predicted by Model 10 for disk center, was therefore convoluted by a Gaussian distribution of macroscopic velocities with an r.m.s. value of $1.5 \mathrm{~km} \mathrm{~s}^{-1}$. Figure 4 shows the excellent

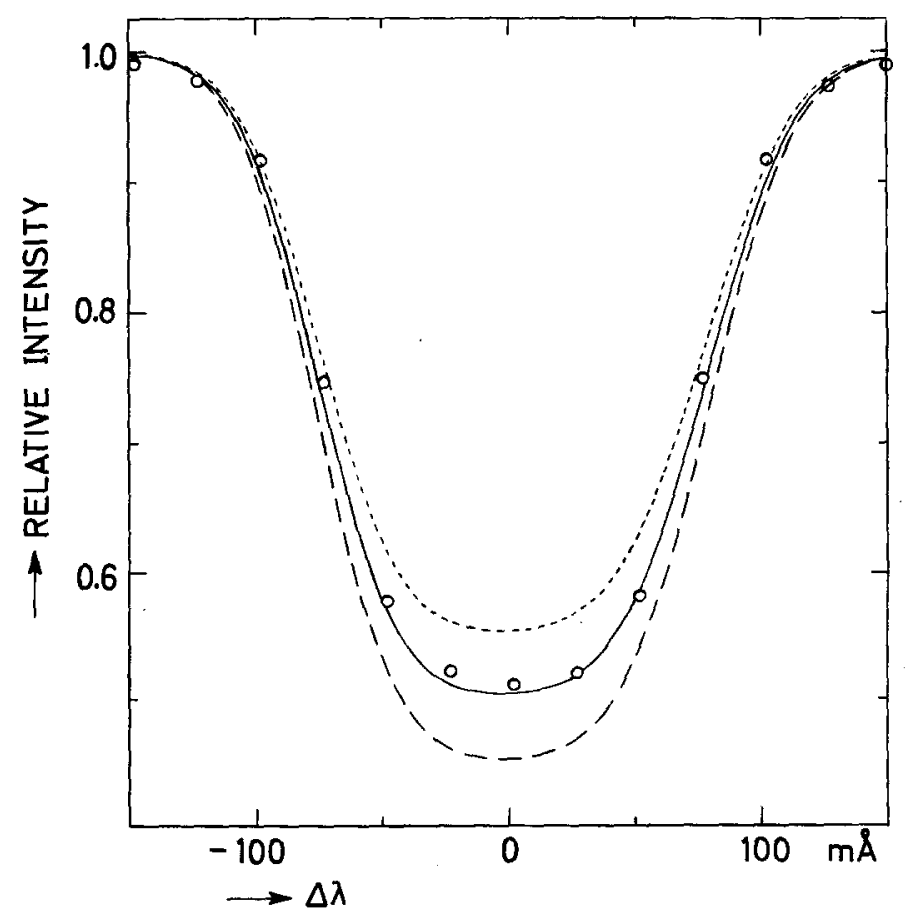

Fig. 4. Profile of Mn 5395 as predicted by Model 10 (solid line) and observed at Kitt Peak for disk center (open circles). Steeper and flatter models give dashed and dotted lines, respectively. 
agreement of the predicted profile with the observations of Kitt Peak (Testerman, 1973). The shape of the line profile mainly confirms the correctness of the hyperfine structure pattern used for the calculation of equivalent widths.

\section{Modelling Granular Temperature Gradients}

We now demonstrate the application of our procedure to model granular temperature gradients. In order to fit the observed variation of continuum and lines in the granulation (Elste, 1985b) a smaller change of the gradient was required in the layers where the manganese line is formed. A larger gradient variation was indicated for the deeper photospheric layers, in which continuum and the carbon line are formed. The resulting models are shown in Figure 5. The corresponding profiles of the manganese line are included in Figure 4 . The equivalent width of this line varies by $12 \%$ around the average, i.e., Model 10. The continuum for the steeper model is only $7 \%$ above that of Model 10 , while the carbon line equivalent width is up by fifteen percent. It must be mentioned here that the observations have not yet been corrected for effects of seeing and telescopic stray light due to lack of funding. Nevertheless we have demonstrated how well this procedure is suited for measuring temperature gradients in the solar granulation. The observing technique and detailed analysis will be presented in a forthcoming paper.

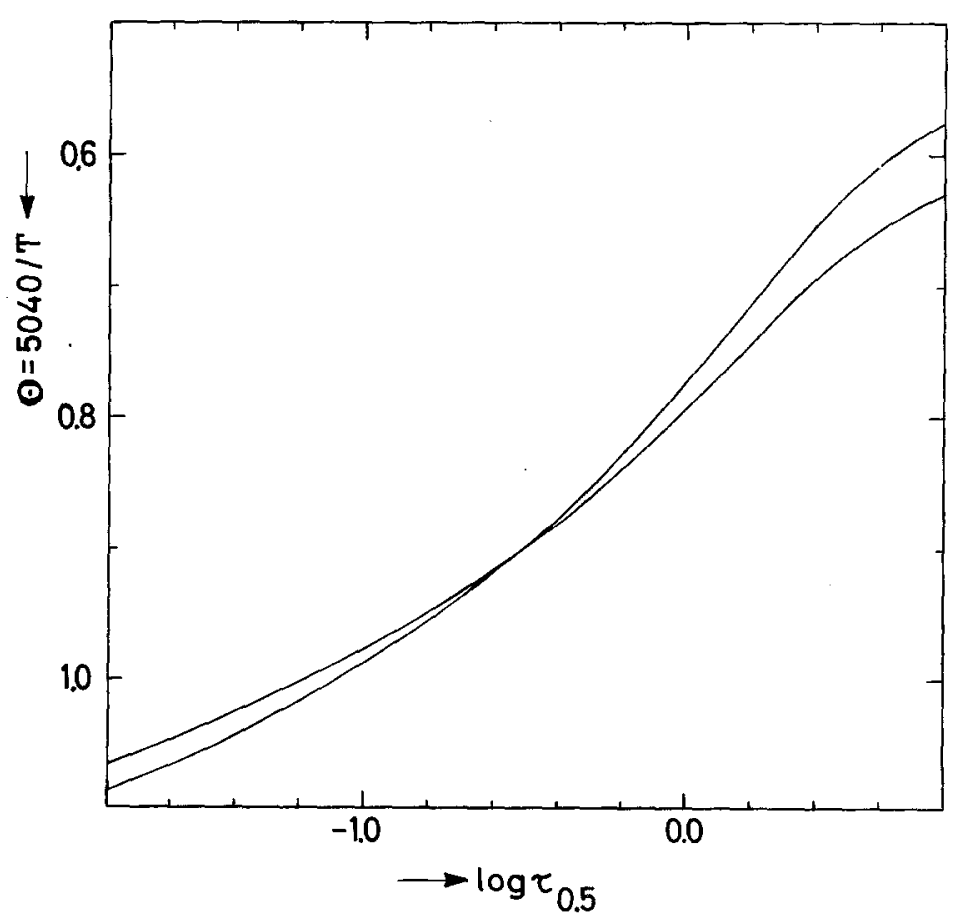

Fig. 5. First approximation temperature models for the granulation: steeper for granule centers, flatter for intergranular lanes. 


\section{Conclusion}

The equivalent widths of $\mathrm{C}_{\mathrm{I}} 5380$ and $\mathrm{Mn}$ I 5395 prove to be sensitive indicators for spatial and depth variations of the temperature structure in photospheric heterogeneities. In particular both lines increase or decrease with steeper or flatter than average temperature gradients. It is evident that the deviation of equivalent widths from the average will have to be interpreted as variations in temperature gradients. The main source of error will thus be the washing out of real variations by the seeing. For the general behavior we have compared the center-to-limb variation of the set of manganese lines of various excitation and of the high excitation carbon line with model predictions. We find that the best fit is obtained for a homogeneous model photosphere which also describes the limb-darkening of the continuum in the spectral range of the lines.

Let us consider a homogeneous model atmosphere to be a reasonable approximation of the complex structured heterogeneous photosphere. The limb-darkening of the continuum as well as the center-to-limb variation of the equivalent widths of weak lines in the same spectral region then reflect the average temperature distribution. In this case it is Model 10 or its modification MODFD.10, which should be chosen as a reference for analyzing variations of temperature gradients in the granulation. The same reference model should be used to analyse the time-variations of the average temperature of the disk at the depth level of line formation. As has been shown by Elste (1985b), we must expect the temperature in the layers in which the manganese line 5395 is formed to be higher than average in those regions on the Sun where we also find magnetic fields. Detailed observational procedures and results of the temperature structure in the solar granulation will be presented in a forthcoming paper.

\section{Acknowledgement}

The author likes to thank Dr C. R. Cowley for helpful comments on the manuscript.

\section{References}

Brix, P. and Kopfermann, H.: 1952, Landolt-Börnstein Springer-Verlag, Berlin, Vol. I, part 5.

David, K. H. and Elste, G.: 1962, Z. Astrophys. 54, 12.

Elste, G.: 1955, Z. Astrophys. 37, 183.

Elste, G.: 1968, Solar Phys. 3, 106.

Elste, G.: 1969, Astron. Astrophys. 3, 277.

Elste, G.: 1985a, in R. Muller (ed.), High Resolution in Solar Physics, Springer-Verlag, Berlin, p. 169.

Elste, G.: 1985b, in H. U. Schmidt (ed.), Workshop on Theoretical Problems in High Resolution Solar Physics,

Max-Planck-Inst. für Astrophysik, München, p. 185

Elste, G. and Teske, R. G.: 1977, Solar Phys. 58, 275.

Gingerich, O. J., Noyes, R. W., Kalkofen, W., and Cuny, Y.: 1971, Solar Phys. 18, 347.

Livingston, W:: 1985, private communication.

Petro, L. D., Foukal, P. V., Rosen, W. A., Kurucz, R. L., and Pierce, A. K.: 1984, Astrophys. J. $283,426$.

Pierce, A. and Slaughter, C.: 1977, Solar Phys. 51, 25.

Testerman, L.: 1973, private communication.

Unsöld, A.: 1955, Physik der Sternatmosphären, Springer-Verlag, Berlin.

Vernazza, J. E., Avrett, E. H., and Loeser, R.: 1976, Astrophys. J. Suppl. 30, 1.

Wittmann, A.: 1980, Astron. Astrophys. 83, 312. 